\title{
INVESTIGACION
}

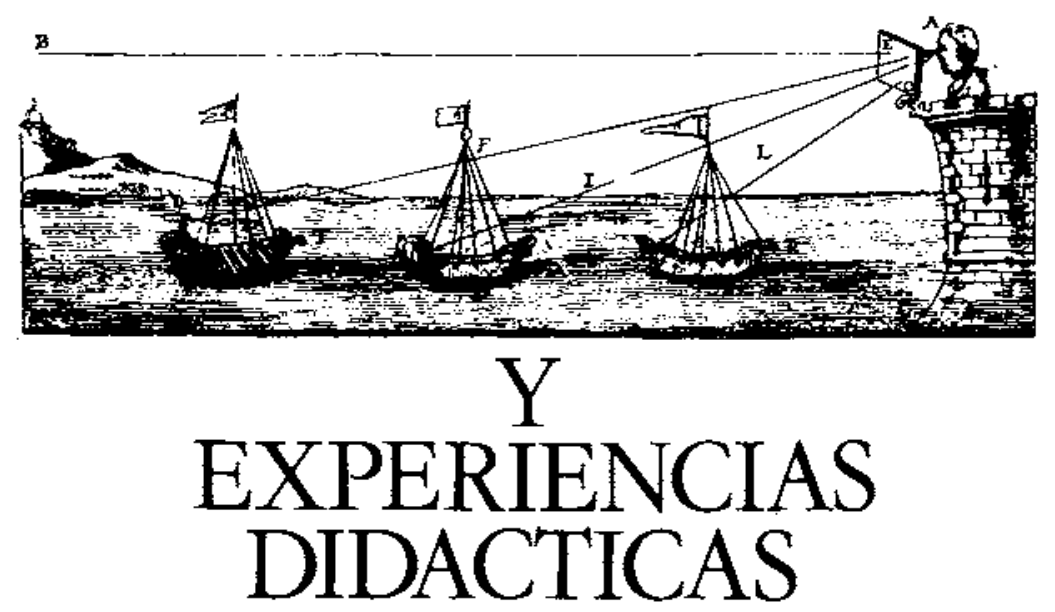

\section{PARTICIPATION DES MAÎTRES AUX MODES DE RAISONNEMENT DES ÉLEVES}

VIENNOT, L. et KAMINSKI, W.

L.D.P.E.S. (Laboratoire de Didactique de la Phyṣique dans l'Enseignement Supérieur, Université Paris 7, Tour 23, 2 Place Jussieu, 75251 Paris Cedex 05)

Conférence présentée au troisième Congrès International sur la recherche en didactique des Sciences et des Mathématiques à Saint Jacques de Compostelle - Septembre 1989.

\section{SUMMARY}

This paper analyses the relationship between teachers and students' reasoning.

\section{INTRODUCTION}

Une longue tradition de pensée et plusieurs courants de recherche contribuent à souligner les différences entre les compétences intellectuelles du maître et celles de I'élève. Ainsi la distinction entre la connaissance commune et la connaissance scientifique dans la tradition bachelardienne (Bachelard 1938), celle que l'on établit entre novices et experts dans les recherches sur la résolution de problème, contribuent-elles à accréđiter l'idée d'une rupture de statut de la connaissance et des modes de fonctionnement cognitifs entre I'élève et le mâttre. 
Je chercherai ici à équilibrer ce point de vue, plutôt qu'à le combattre à outrance, en soulignant la continuité qui existe entre les raisonnements des maitres et ceux des élèves, et en esquissant une analyse des causes de ce fait. Les faits expérimentaux qui viennent à l'appui de ce que j'avance n'ont pas tous un caractère bien orthodoxe, car les maîtres n' aiment pas être l'objet d'une investigation. Plutôt que des résuitats d'enquête en bonne et due forme, il peuts'agir de sondages informels, d'analyses d'ouvrages scolaires ou de vulgarisation considérés comme bons par de nombreux enseignants, de réactions d'enseignants lors de stages de formation en physique ou en didactique, ou même d'analyses de textes rédigés par des chercheurs en didactique.

Cette réflexion débouche sur des questions portant sur nos objectifs d'enseignement.

\section{LES ENSEIGNANTS ET LES RAISONNE- MENTS NATURELS}

Un premier facteur de rapprochement entre élèves et maîtres est à rattacher à ce que l'on a appelé, en manière de raccourci, les raisonnements naturels. Dans l'important courant d'études portant sur les idées des élèves et des étudiants en physique, plusieurs investigations (Closset 1983 et Rozier) portant sur des étudiants avancés ont fait apparaître des erreurs de même nature que chez des élèves plus jeunes, et ceci avec des taux importants. Les erreurs analogues trouvées dans les manuels renforcent souvent l'impression que la difficulté correspondante est mal connue et probablement très inégalement dominée par les enseignants. La mécanique, en particulier la Ioi des actions réciproques (Viennot 1982), l'électrocinétique encore davantage (Closset 1983), ont été l'occasion de telles constatations.

Le point important qui en ressort est le suivant: ce qui demeure à un niveati élevé de formation, ce ne sont pas les réponses erronées à des questions proposées à des élèves plus jeunes. Pratiquement aucun étudiant en fin d'études, aucun enseignant, ne dira que, si deux ampoules identiques encadrent une résistance, la deuxième brille moins fort que la première (Encadré I, question 1). Mais si l'on s'intéresse au type de raisonnement qui préside à cette erreur, un raisonnement «séquentiel» où un objet -ici le courant-subit des aventures successives sans action de l'aval sur l'amont, alors on observe que ce raisonnement demeure et se manifeste chez les «experts» dès qu'ils sont confrontés à une question nouvelle pour eux (Encadré I, question 2).

La perception de la continuité entre les difficultés de l'élève et celles du maître est donc liée à la mise à jour de formes de raisonnement relativement générales, rendant compte de familles de question-réponses intervenant sur des contextes physiques variés. Un simple catalogue d'erreurs communes donne, au contraire, 1'illusion d'une discontinuité entre élèves et maîtres, ces derniers se révélant beaucoup pius performants que les premiers sur de nombreuses questions.

ENCADRÉ I (Réf. 2) Diverses situations suscitant des raisonnements séquentiels:

* E1: élèves de fin d'ensetgnement secondaire belge. $N \cong 50$

E2: étudiants de première année universitaire. $N \equiv 80$

E3: étudiants ayant eu deux à quuatre ans d'enseignement supérieur. $N \equiv 70$

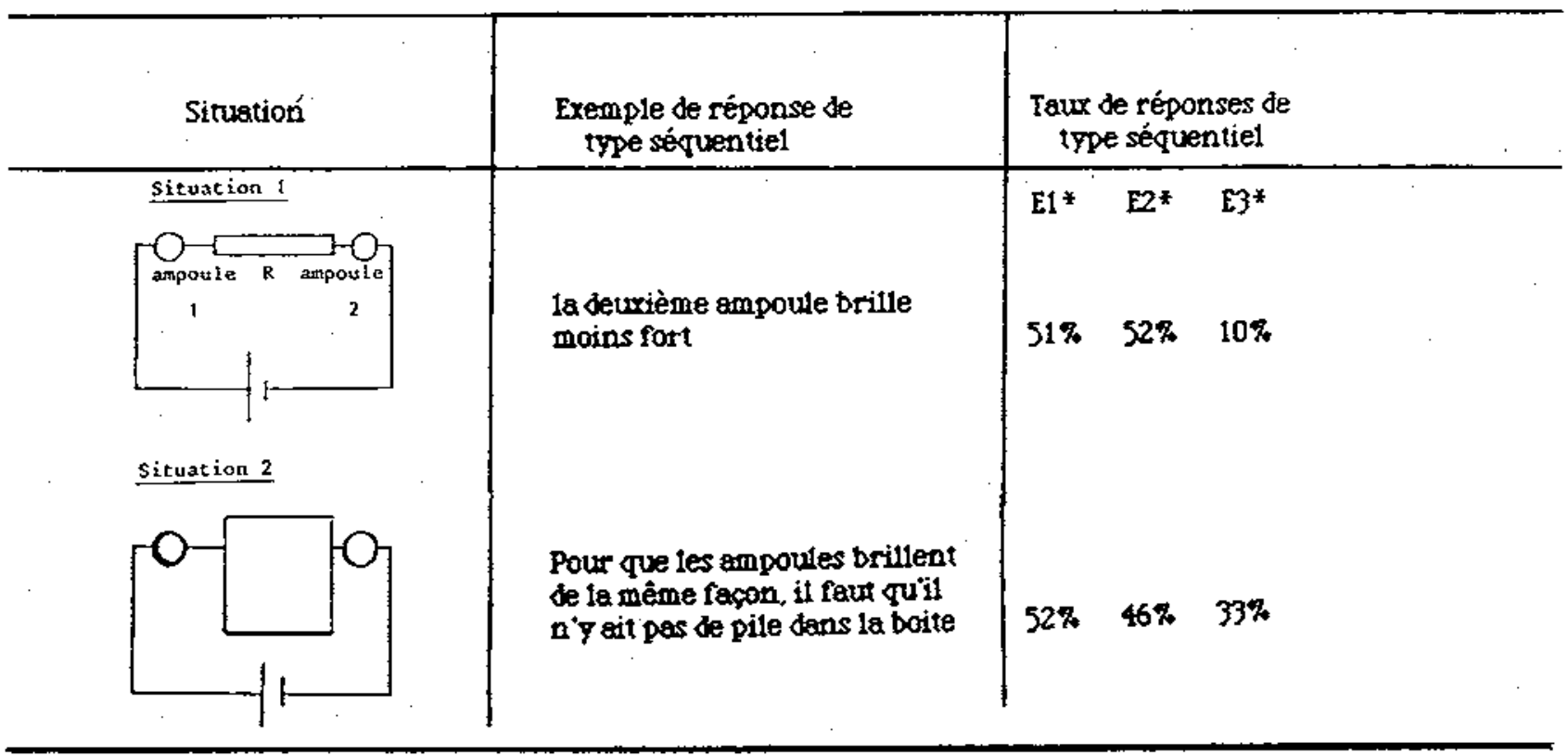


Je vais donc illustrer cette continuité de difficultés sur l'axe des compétences scolaires par un aspect du raisonnement non lié à un contenu particulier de la physique: la tendance à la "réduction fonctionnelle» dans le traitement des problèmes à plusieurs variables, et plüs largement, le «raisonnement linéaire causal» (termes définis plus loin). Ce sera.l'occasion de voir que les difficultés des enseignants ne se manifestent pas nécessairement par des erreurs, mais peuvent avoir d'autres conséquences.

\section{CONTINUITÉ ENTRE ÉLÈVE ET MÂ̂- TRE: EXEMPLES DE DIFFICULTES DANS LE TRAITEMENT DES PROBLEMES A PLU- SIEURS VARIABLES}

Un regard rapide sur les programmes d'enseignement secondaire, par exemple en France, pourrait laisser penser que nos élèves sont habitués à traitèr des problèmes à plusieurs variables. En fait, ils sont essentiellement habitués à utiliser, pour des calculs numériques, des formules où figurent plusieurs lettres. Ce n'est que rarement qu'ils sont sollicités à faire de ces formules une analyse fonctionnelle du type "si telle grandeur augmente, et si telle et telle autres restent constantes, alors telle grandeur...». Le résultat d'enquête résumé en encadré il est là pour en témoigner. Plusieurs études (Viennot et Rozier 1982) confirment largement cette constatation.

Pourtant il y a là à la fois un enjeu fondamental pour l'éducation du raisonnement scientifique (au sens large) et une difficulté considérable: la manipulation de plusieurs variables est complexe, et l'impossibilité de raisonner sans précaution sur un seul couple de variables est difficile à admettre.

Pour illustrer la continuité de difficulté qui réunit maîtres et élèves sur ces points, nous prendrons ici des exemples en thermodynamique. Mais ìl est bien clair que le phénomène déborde largement ce domaine typique de la physique, et mêrne la physique tout court: que l'on songe seulement à l'économie...

Les procédures couramment mises en cuvre pour échapper à la complexité des problèmes à plusieurs variables peuvent grossièrement se classer en deux catégoties: réduction du nombre des variables prises en compte, ou bien traitement simplifié du jeu, complet cette fois, des variables pertinentes.

\section{La réduction fonctionnelle}

Illustrons d'abord la première, que l'on désignera par la rubrique «réduction fonctionnelle». Le résultat évoqué en encađré II en relève bien évidemment, ainsi que ceux, par exemple, évoqués par J. Piaget (1941) à propos de la relation $L=V T$, ou ceux rapportés par $L$. Maurines (1988) à propos đe la même telation appliquée à la propagation de signaux mécaniques: une relation entre trois variables est traitée en ignorant I'une d'entre elles. «Ignorer» est le terme adapté chaque fois que rien n'est dit au sujet de la dite variable; par exemple: «le mobile qui va plus vite va plus loin" (sans que l'on ne sache rien sur la durée). Un cas intermédiaire est celui où l'élève dit explicitement que cette troisième variable est constante. Même Iorsqu'il s'agit d'une erreur, il y a cette fois prise en compte de toutes les variables.

Une telie prise en compte n'est nullement systématique chez les élèves. De ce point de vue, il apparaît que les enseignants ont une tolérance considérable envers les expressions raccourcies, décrivant ou expliquant les phénomènes de manière tronquée. Ils pratiquent euxmêmes volontiers ce mode d'expression dès qu'il quittent le langage formel propre à la mathématisation, et qu'ils souhaitent "faire comprendre» quelque chose à leurs élèves ou énoncer verbalement une propriété physique. Deux exemples:

Considérons l'énoncé suivant de la loi de Joule: «L'énergie interne d' une masse donnée de gaz parfait ne dépend pas de son volume». Lors d'une enquête menée par S. Rozier

\section{ENCADRE II}

Une explication de texte proposée à des étudiants de première année universitaire ( $\mathrm{N}=25$ ) (à propos d'un test de J.L. Closset, réf. 2).

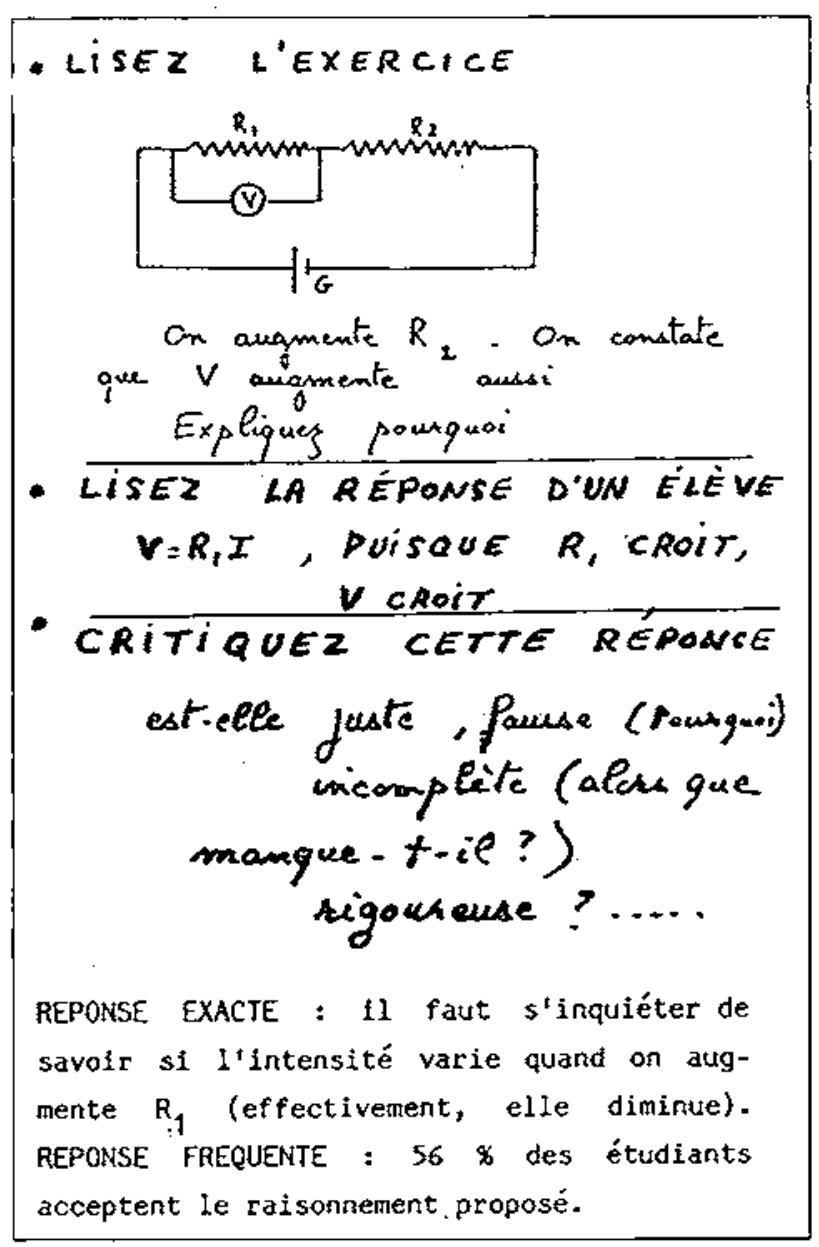


(1982), cet énoncé a été déclaré complet, acceptable et compréhensible par $45 \%$ d'un échantitlon de 45 enseig-' nants français universitaires et/ou agrégés ${ }^{1}$. Cet énoncé ou bien n'a pas de sens, ou bien est faux, si l'on n'ajoute pas «à température fixée». Chez ces enseignants, la précision vient d'elle-mêrne dès le passage à l'écriture formelle $\delta U / \delta \mathrm{V}_{T N}$. Ce garde-fou, ils l'ont bien appris. Mais leur tolérance indique qu'ils sous-estiment les pièges que tend le langage verbal, pour peu qu'on le prenne au sérieux. Ainsi ce deuxième exemple:

Un ouvrage de vulgarisation (Maury 1989) fort bien accueilli en milieu universitaire écrit, à propos de l'épaisseur des hublots d'un avion: «les molécules de l'air sont beaucoup moins nombreuses que près du sol et donc la pression extérieure de l'air extérieur sur la vitre est beaucoup plus faible qu'au niveau du sol». Proposé à la critique d'enseignants en formation en physique (Paris, 1986-87, premier cycle du secondaire, $N=30$ ) ou en didactique (tous niveaux, 1989, Paris, $N=20$, Milan $\mathrm{N}=25$ ), cet énoncé est quasi unanimement accepté. Mais que comprendre lorsque le même ouvrage, évoquant la montgolfière cette fois, nous laisse entendre qu'il y a «moins de molécules» aussi, et que pourtant la pression, bien évidemment, n'y est pas plus faible qu'à l'extérieur?

Affaire de température, bien sûr. Mais alors pourquoi n'en parle-t-on pas dans le cas de l'avion? Réponse usuelle: "parce que cela ne compte pas" ${ }^{2}$. On évoque donc le facteur dominant sans s'expliquer sur les autres. $S$ 'il s'agit d'un choix pédagogique, il est discutable puisqu'il ne peut que renforcer l'idée qu'il n'y a jamais à la fois qu'un seul facteur qui compte. Mais d'autres résultats suggèrent qu'il peut s'agir autant d'une manifestation d'une tendance très forte du raisonnement naturel que d'un choix pédagogique délibéré et maîtrisé.

\section{Le raisonnement linéaire causal}

Cecí nous amène à évoquer la manière dont $S$. Rozier décrit les grandes tendances du raisonnement devant les problèmes à plusieurs variables (Rozier). Un exemple introduira son analyse en termes de «raisonnement linéaire causal»:

A propos de la dilatation isobare d'un gaz parfait, des étudiants des trois premières années universitaires (toutes catégories indiscernables au niveau des taux de réponses) sont requis d'expliquer le résultat suivant: "on chauffe un gaz parfait sous pression constante. On observe que la température $T$ augmente et que le volume $V$ augmente». Une forte proportion d'entre eux (40\%) fournit une explication qui peut se schématiser ainsi:

\section{Apport de chaleur $\rightarrow T$ augmente $\rightarrow$ (la pression) aug- mente $\rightarrow V$ augmente}

On note dans cette réponse type la linéarité de l'argumentation: contrairement à la réponse correcte, chaque maillon de là chaîne lie une seule variable à une seule autre. Les variables sont bien toutes là cette fois, mais elles sont liées deux à deux seulement. Ce trait apparaît chez les étudiants à propos de multiples questions.
Le deuxième aspect marquant du raisonnement linéaire causal émerge notamment à travers cette contradiction entre la donnée de I'énoncé - $\varepsilon$ chauffage est isobare-et un élément de la réponse: "p augmente». La contradiction s'estompe lorsqu'on comprend que l'argumentation prend, dans le raisonnement naturel, une connotation chronologique. Chaque flèche $« \rightarrow$ » signifie «donc», mais aussi «ensuite». Les termes français «alors» ou anglais «then» amalgament d'ailleurs parfaitement les deux significations. Les événements évoqués dans la chaîne d'implications sont implicitement, et parfois explicitement, considérés comme successifs, et donc comme temporaires. Dans ce raisonnement, les choses sont comprises ainsi: dans un premier temps, le volume est bloqué, alors il est clair que I'apport de chaleur élève température (aucun travail n'est fourni à l'extérieur) et pression (seule la vitesse moyenne des particules varie, non leur concentration); puis, dans un deuxième temps, le piston est relâché, alors la pression reprend la valeur extérieure tandis que le volume augmente.

Tout se passe comme si l'analyse à une seule variable,ou encore en "phénomène dominant», imposait sa rigidité au raisonnement et n'autorisait, comme adaptation à la complexité du phénomène, qu'une séquentialisation chronologique.

L'analyse des problèmés évoqués en termes exclusifs de "phénomènes dominants», l'absence quasi systématique de prise en compte simultanée de phénomènes différentsetéventuellementcompétitifs, sont volontiers présentés comme un choix pédagogique de simplicité.

II faut être conscient qu'un tel choix entre en résonance avec une tendance extrêmement forte et générale (voir les résuitats de Rozier) à laquelle les maîtres n'échappent pas.

Dans cette tendance, la distinction entre la chronologie effective des événements et celle de l'argumentation est très floue, les cheminements intellectuels en matière de temps sont très peu explicités. Ce que I'on observe, c'est une mise en forme préférentielle d'explications en termes d'histoires où se déroulent des événements simples et successifs. Des victimes désignées de ce traitement sont par exemple les régimes permanents de non équilibre. L'exemple-type est celui de la serre: combien d'enseignants, de brochures de vulgarisation ne donnent-ils pas une explication qui se résume à celle-ci: le rayonnement solaire est piégé, ou bien: il rentre plus d'énergie qu'il n'en sort, sans réaliser qu'ils évoquent au mieux le phénomène dominant du régime transitoire pour expliquer le régime permanent, ni que le phénomène qu'ils évoquent ne saurait durer sans danger pour le jardinier.

Dernier exemple pour signaler combien étudiants et enseignants partagent une faible sensibilisation à l'analyse des régimes permanents. Des enseignants en formation en didactique (1989, Paris $\mathrm{N}=20$, Milan $\mathrm{N}=25$ ) ont été sollicités pour proposer une réponse du maître au commentaire suivant, recueilli par Rozier à propos d'une compression adiabatique, et très fréquent chez les étudiants universitaires: «les chocsentre molécules à l'intérieur du gaz dégagent de la chaleur». Aucun de ces maîtres n'a 
proposé une remarque centrée sur l'idée de régime permanent, ni souligné qu'un tel régime est évidemment incompatible avec une production continue de chaleur en récipient adiabatique. Un tel argument, dont $S$. Rozier montre bien qu'il porte juste sur un noud du raisonnement naturel, n'est pas spontanément utilisé probablement parce que les maîtres partagent, fût-ce à un moindre degré, cette composante du raisonnement naturel qu'est I'inconscience et le flou sur la localisation dans le temps des événements évoqués, leur éventuelle simultanéité, leurs conséquences dans la durée.

\section{ENSEIGNEMENT SCOLAIRE: EFFET BOOMERANG SUR LES MAITRES. EXEM- PLES EN OPTIQUE}

Les difficultés évoquées ci-dessus, probabiement en résonance avec, 'sinon amplifiées par, l'enseignement reçu, relèvent pourtant a priori du registre «raisonnement naturel». Largement interdisciplinaires et répandues, au moins dans notre culture occidentale, elles précèdent et débordent amplement le cadre scolaire.

Je voudrais évoquer un second type de rapprochement entre élèves et maîtres qui relève essentiellement, me semble-t-il, de l'acquis scolaire. C'est un exemple d'optique qui m'y aidera.

L'encadré IIIa présente une question proposée par $\mathrm{W}$. Kaminski (1986) à diverses populations françaises de find'enseignement secondaire $\left(\mathrm{N}_{\text {total }}=170\right)$ et d'enseignants (expérimentation informelle) et reprise par l'auteur auprès d'enseignants espagnols en formation en didactique (Madrid $1988 \mathrm{~N}=35$, expérimentation informelle). Seuls figurent sur la feuille un objet rectiligne $A B$ et une image rectiligne $A^{\prime} B^{\prime}$ non parallèle à $A B$. On demande de trouver l'emplacement de la lentille sur le dessin. Dans aucune de ces populations le taux de réponses justes ne dépasse 5\%. Le fait important est l'appel massif, à tous les degrés de compétence, à la panoplie d'outils de construction classiques: centre optique, certes, mais aussi axe optique (préférentiellement horizontal), foyers primaires ou secondaires, rayons parallèles...On trouve en IIIb un exemple des dessins qui en résultent.

En fait, il suffit pour répondre de prendre au sérieux la définition de l'image ( $A^{\prime}$ ) d'un point (A) dans le stigmatisme gaussien: tout rayon issu de $\mathrm{A}$ et rencontrant la lentille a ensuite pour support une droite qui passe par $A^{\prime}$. Donc le rayon incident $A B$ émerge selon $A^{\prime} B^{\prime}$. Nul aigorithme de résolution habituel, donc, mais seulement l'appel au concept même d'image.

Ici, iI apparaît clairement que c'est le centrage trop exclusif de l'enseignement sur les questions et constructions traditionnelles -position de l'image?, rayons de constructions, etc.- qui bloque une réflexion plus conceptuelle et interdit l'accès à la solution. L'absence de telles questions dans l'enseignement que ces maîtres ont éventuellement donné les a dispensés de voir les limites de celui qu'ils ont reçu.

\section{ENCADRÉ III}

a) od̀ est la Ientille?

b) une réponse d'enseignant

c) éléments pour la réponse correcte

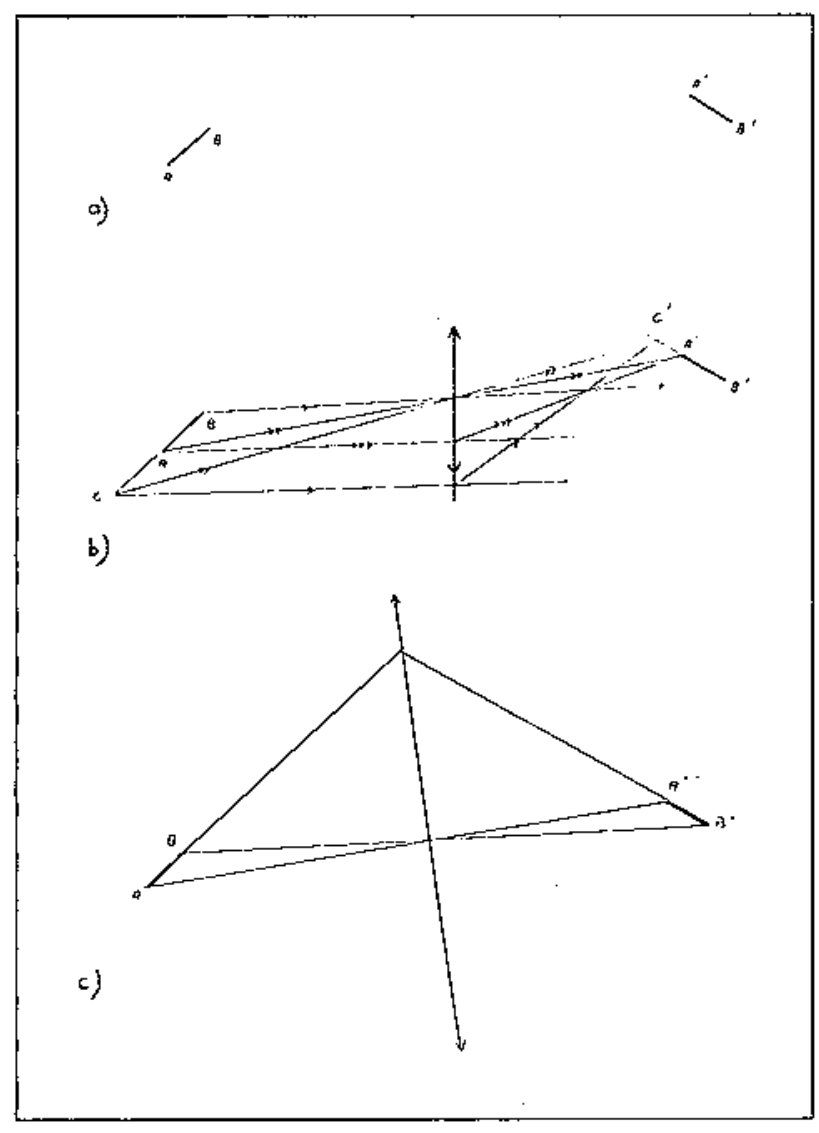

Encore en amont de la notion d'image, celles de lumière et d'objet diffusant sont I'occasion du même type de constat. Les résultats de W. Kaminski (1989), portant sur environ 50 professeurs du premier cycle du secondaire en formation continue, confirment à quel point la pauvreté conceptuelle des objectifs et des activités scolaires usuels, la connaissance des réponses à quelques questions, peuvent cacher aux enseignants eux-mêmes leurs propres lacunes dans la compréhension de ces concepts fondamentaux de l'optique. Ajoutons qu'une fois ces lacunes explicitement prises en compte comme objet du travail de formation ${ }^{3}$, les enseignants concernés répondent notoirement mieux à la question ci-dessus (Encadré III) que des enseignants du second cycle du secondaire dont ia formation initiale en physique est en moyenne supérieure, selon les critères classiques.

On n'incriminera pas ici le raisonnement naturel, mais bien I'illusion scolaire qui consiste à penser que l'apprentissage de procédures algorithmiques suffit et entraîne derrière lui la compréhension des concepts.

L'illusion, on vient de le voir, permet d'ignorer sinon d'entretenir des lacunes conceptuelles. Il arrive que cet 
effet négatif aille plus loin: certains stéreotypes scolaires non seulement dissimulent une compréhension incomplète, mais aussi engendrent des idées fausses. Les résultats de l'enquête de Fawaz (1985) au Liban, largement confirmés par des sondages informels auprès d'enseignants en formation (W. Kaminski, 1988-89 Paris, $\mathrm{N}=50$; L.Viennot, 1988 Madrid, $\mathrm{N}=35$ ) montrent qu'une majorité d'enseignants pense, à tort précisons-le, qu'une image réelle ne peut se voir à l'oeil nu. Là encore, il s'agit d'un effet de I'enseignement et non du raisonnement naturel. D'ailleurs les élèves interrogés par A. Fawaz, moins influencés sans doute par l'enseignement que leurs maittres, donnent en majorité une réponse correcte (mal justifiée).

\section{DISCUSSION}

Les éléments que I'on vient de citer soulignent ce qui, paradoxalement, rapprochent élèves et maîtres dans leur compréhension de la physique. Comme d'habitude, ce sont les difficultés ou les lacunes qui ont servi ici de détecteurs privilégiés de cette proximité, mais rien n'autorise à penser que celle-ci ne s'étend pas à certaines facilités ou à certains succès.

Dans ce rapprochement, deux composantes ont été évoquées: le raisonnement naturel et l'effet de l'enseignement qu'ils donnent sur les maîtres eux-mêmes. Présentées de manière contrastées pour les besoins de la démonstration, ces composantes s'imbriquent, fusionnent, ou entrent en résonance l'une avec l'autre, la plupart du temps.

L'intérêt d'une telle constatation n'est évidemment pas d'entamer l'idée que les maîtres en savent beaucoup plus que leurs élèves. Ceci est incontestable. Il ne s'agit pas non plus de nier que beaucoup d'entre eux échappent à Ia description faite ici.

Mon but est plutôt de donner, à travers ce rapprochement, de I'intérêt aux aspects conceptuels qui en sont la matière. Ces aspects qui nous rapprochent de nos élèves méritent l'attention, quelque soit le degré auquel nous les maîtrisons. Ils nous interrogent, me semble-t-iI, sur ce que nous pouvons souhaiter faire comprendre à nos étudiants, et, corrélativement, sur la nature des activités à leur proposer.

\section{Revenons aux exemples ci-dessus.}

On sait bien ce qui justifie les raisonnements sur une seule variable: idéal expérimental («toutes choses égales par ailleurs»), nécessité impérative de simplification de la réalité physique pour la comprendre et pour la faire comprendre progressivement à de jeunes esprits, enfin nécessité de raccourci dans le discours entre pairs.

La question est maintenant: cela vaut-il la peine de chercher à concilier ces démarches simplificatrices -d'expérimentation d'une part, d'exposition d'autre partavec le souci de ne pas laisser subsister indéfiniment les rigidités du raisonnement linéaire causal: oubli de variables pertinentes, pseudo-explications qui décrivent sans la situer par rapport aux autres la «cause dominante», déficience dans la compréhension des systèmes avec retroaction, des régimes permaments de non équilibre, etc.

Ce n'est pas ici qu'il fait aborder le «comment» d'une telle entreprise, d'ailleurs déjà en chantier ici ou là ${ }^{4}$. J'ai seulement voulu souligner à la fois l'importance de l'enjeu et la nécessité de prendre, dans notre évaluation des obstacles à prévoir, une certaine distance avec nos habitudes de pensée d'enseignants, pour affronter rationnellement les difficultés du raisonnement naturel, sans les surestimer non plus: après tout, l'éducation du raisonnement à plusieurs variables peut commencer avec des objets aussi simples que la surface d'un rectangle.

L'exemple de l'optique nous confronte à des faits moins conséquents, mais néanmoins significatifs. Il n'est pas en soi bien grave que des enseignants pensent ne pas pouvoir voir directement une image réelle. Mais il apparâ̂t à travers ce fait que l'enseignement classique donné par ces enseignants ne les amène pas à comprendre vraiment ce qu'est une image ní le phénomène de la vision. Comment espérer qu'ils y conduisent leurs élèves? En revanche, si une telle compréhension devient partie intégrante des buts de l'enseignement, les activités de type algorithmique, rayons de construction à l'appui, doivent être complétées d'autres beaucoup plus éprouvantes conceptuellement. En l'occurrence, W. Kaminski (1989) a mis au point et évalué sur des enseignants un ensemble de telle activités de formation. De son travail, il ressort une fois de plus que si l'on cherche à atteindre les concepts mêmes, ici ceux d'image et de vision, un sujet apparemment bien mince, le miroir par exemple, s'épaissit considérablement tout en prenant de l'intérêt.

De 1'intérêt: peut-être est-ce là le maître-mot qui doit ressortir, avec celui de vigilance, de ce constat de difficultés communes aux enseignants et aux élèves.

La vigilance s'impose à nous, enseignants, dans le repérage des difficultés des élèves, lesquelles risquent de nous échapper d'autant plus que nous les partageons, ou bien d'être, de ce fait, mal évaluées.

L'intérêt vient de ce que les obstacles sur lesquels les raisonnements des élèves et ceux des maîtres se rejoignent signalent en général une matière véritablement consistante sur le plan conceptuel. Ces points où bute la pensée sont donc, au moins, à prendre en considération pour le choix de nos objectifs d'enseignement et l'élaboration des activités à proposer à nos élèves.

\section{NOTES}

(1) L'agrégation est le concours de recrutement de professeurs du secondaire qui a le niveau le plus élevé.

(2) Pourtant notons au passage que la température extérieure aux altitudes de croisière courantes $(\sim 10 \mathrm{~km})$ peut être de l'ordre de 
$-70^{\circ} \mathrm{C}$, soit un écart de l'ordre de $25 \%$ par rapport au niveau du sol.

(3) C'est le sujet de l'atelier proposé par W. Kaminski à ce congrès.

\section{REFERENCES}

BACHELARD, G., 1938. La formation de l'esprit scientifique. (P.U.F., Paris, 1970).

CLOSSET, J.L., 1983. Le raisonnement séquentiel en éléctrocinétique. Thèse non publiée, Université Paris 7.

FAWAZ, A. et Viennot, L, 1985. Image optique et vision: enquête en classe de première au Liban, Bulletin de l'Union des Physiciens, 686, pp. 1125-1146.

KAMINSKI, W., 1986. Statut du schéma par rapport à la réalité physique: un exemple en optique. Mémoire de D.E.A., Université Paris 7, sur demande au L.D.P.E.S.

KAMINSKI, W, 1989. Conceptions des enfants (et des autres) sur la lumière, Bulletin de l'Union des Physiciens, 716.

MAURINES, L. et SALTIEL, E., 1988. Mécanique spontanée du signal, Bulletin de l' Union des Physiciens, 707, pp. 10231041 .
(4) Je pense, par exemple, à la recherche didactique italienne en matière d'enseignement élémentaire, notamment sur le thème de la thermodynamique: P. Guidont, E. Giordano, M. Vicentini-Missoni... Ex.: Calore e temperature, E. Giordano, C. Longo, P. Majorino Bonelli. Emme Edizione. Turin.

MAURY, J.P., 1989. La glace et la vapeur, qu'est-ce que c'est?, (27) Paiais de la découverte: Paris.

PIAGET, J. et. INHELDER, B., 1941. Le développement des quantités physiques chez l'enfant, Delachaux-Nestlé, Neufchatel.

ROZIER, S., 1982. L'implicite en physique: les étudiants et les fonctions de plusieurs variables. Mémoire de D.E.A. Université Paris 7 , sur demande an L.D.P.E.S.

ROZIER, S., Le raisonnement linéaire causal en thermodynamique classique élémentaire. Thèse non publiée, Université Paris 7.

VIENNOT, L., 1982. L'Action et la Réaction sont-elles bien (Égales et) opposées? Bulletin de l'Union des Physiciens, 640 , pp. 479-488.

VIENNOT, L. L'implicite en physique: les étudiants et les constantes, European Journal of Physics, 3, pp. 174-180. 